\title{
Gene expression profiles are different in venous and capillary blood: implications for vaccine studies
}

\author{
${ }^{1}$ Stein DF, ${ }^{2} \mathrm{O}$ 'Connor $\mathrm{D}^{*},{ }^{2} \mathrm{Blohmke} \mathrm{CJ}$, ${ }^{2}$ Sadarangani $\mathrm{M}$, ${ }^{2}$ Pollard AJ
}

Affiliations:

${ }^{1}$ School of Clinical Medicine, University of Cambridge

${ }^{2}$ Oxford Vaccine Group, Department of Paediatrics, University of Oxford, and the NIHR Oxford Biomedical Research Centre, Oxford, United Kingdom.

*Corresponding Author: Daniel O’Connor, Oxford Vaccine Group, Department of Paediatrics, Centre for Clinical Vaccinology \& Tropical Medicine (CCVTM), The Churchill Hospital, Old Road, OX3 7LE, United Kingdom.

Tel/Fax: +44 (0)1865 857420

Email: daniel.oconnor@paediatrics.ox.ac.uk 


\section{Abstract}

2 Background: Detailed analysis of the immunological pathways leading to robust

3 vaccine responses has become possible with the application of systems biology,

4 including transcriptomic analysis. Venous blood is usually obtained for such studies

5 but others have obtained capillary blood (e.g. finger-prick). Capillary samples are

6 practically advantageous, especially in children.

7 Methods: The aim of this study was to compare gene expression profiles in venous

8 and capillary blood before, 12 hours and 24 hours after vaccination with 23-valent

9 pneumococcal polysaccharide or trivalent inactivated seasonal influenza vaccines.

10 Results: Gene expression at baseline was markedly different between venous and

11 capillary samples, with 4940 genes differentially expressed, and followed a different

12 pattern of changes after vaccination. At baseline, multiple pathways were upregulated

13 in venous compared to capillary blood, including transforming growth factor-beta 14 receptor signalling and toll-like receptor cascades. After vaccination with the 15 influenza vaccine, there was enrichment for $\mathrm{T}$ and NK cell related signatures in 16 capillary blood, and monocyte signatures in venous blood. By contrast, after 17 vaccination with the pneumococcal vaccination, there was enrichment of dendritic 18 cells, monocytes and interferon related signatures in capillary blood, whilst at 24h 19 there was enrichment for $\mathrm{T}$ and NK cell related signatures in venous blood.

20 Conclusions: These data show differences between venous and capillary gene 21 expression both at baseline, and post vaccination, which may impact on the 22 conclusions regarding immunological mechanisms drawn from studies using these 23 different sampling methodologies.

Keywords: Genomics; Vaccines; Systems Biology; Immunity. 
Vaccines are an unrivalled intervention in terms of impact on morbidity and mortality due to infectious disease, but the immunological processes determining protection following vaccination are not fully understood [1]. The use of high-throughput technology allowing different pathways and their interactions to be examined holistically, and subsequent integration and analysis of the data produced has been used to investigate transcriptional changes in response to vaccination in systems biology analyses. Analysis of such changes have identified gene expression signatures that correlated with immunogenicity following yellow fever and influenza vaccination [2-4].

The comprehensive data thus generated provide a novel approach to interrogate the molecular mechanisms underlying host responses to infection and vaccination and provide a new tool in the development of vaccines. One of the most cost-effective methods of analysing changes in the transcriptome is the use of RNA micro-arrays. Transcriptional data provide a snapshot of the genome wide expression profile at a specific point in time allowing conclusions about the molecular host responses to an immunological stimulus to be drawn if an analysis of global gene expression changes is performed before and after vaccination [2]. While useful, this approach produces thousands of data points, which can make extraction of coherent information difficult. Gene set enrichment, where catalogues of annotated sets of genes are used to interrogate the data, has allowed the extraction of biologically meaningful and objective information, leading to advances in understanding of changes in response to vaccination [5].

Protocols for the exploration of transcriptional changes have not been standardised between studies, sample types, timing and processing. Some studies have used whole blood from venepuncture whereas others use PBMCs from the same type of venous sample and these samples differ significantly [1-3, 6]. It has been suggested that whole blood may result in reduced detection sensitivity, and that prolonged handling of PBMC samples is associated with artifactual changes. Capillary blood has been used as a more convenient sample to obtain, particularly in studies of children. This means that studies using different sample types and processing and analysis methods cannot easily be compared. As gene expression data tend to be susceptible to sample 
71 processing and handling, sampling methods may impact on the conclusions drawn 72 from transcriptomic studies, leading to potentially redundant functional experiments.

73 It is thus important to know whether venous and capillary samples produce results 74 that are comparable. However, the difference in gene expression between capillary 75 and venous blood samples has not been previously studied. In the present study we 76 directly compared data derived from samples obtained simultaneously from 77 individuals by venepuncture (venous) and fingerprick (capillary), which are publically 78 available as a resource from the Gene Expression Omnibus (GEO) database, and 79 investigated differential gene expression before and after vaccination, between these 80 methods [7].

81 


\section{3}

Methods

\section{Data Source}

The raw sample and control data used by Obermoser et al were downloaded from the Gene Expression Omnibus (GEO) database (Accession: GSE30101) [7].

\section{Study design and participants}

The study population has been described in detail previously ${ }^{7}$. Briefly, healthy adult volunteers aged 18-64 years received the seasonal influenza vaccine (Fluzone, Sanofi Pasteur), pneumococcal polysaccharide vaccine (Pneumovax23, Merck) or saline control (Table 1). Blood was obtained at various time points, including four time points where both fingerprick (capillary) and venepuncture (venous) samples were taken simultaneously from the same subject (7 days pre-vaccination [-7d], at the time of vaccination [0d], and 12- and 24-hours post-vaccination [12h, 24h]), providing an opportunity to compare transcript expression between the two sample types.

\section{Table 1:}

\section{Data Processing}

Microarray data were background subtracted, and a force positive modification used so that a $\log _{2}$ transformation could be performed, followed by a robust spline normalisation of the data (R package Lumi) [8]. The data were filtered to only include probes that were detected in greater than $65 \%$ of all the samples in the analysis, to exclude inconsistently detected probes ( $p<0.05$; R Package Genefilter).

\section{Data Analysis: Fold Change}

Paired analysis was conducted to compare transcript expression in capillary and venous blood derived from the same individual. The baseline samples (-7d, 0d) from all study groups - saline, influenza and pneumococcal vaccine groups were analysed. The pre- $(0 \mathrm{~d})$ to post-vaccination fold changes were then tested using a paired t-test between sample types. Comparison of venous against capillary was done using a paired t-test, and pre-, post-vaccination changes were calculated using a linear regression. The changes were compared between venous and capillary with a paired ttest. Only samples with a pair were tested; un-paired samples were not analysed. These tests were fit using the lmFit and eBayes functions (R Package Limma) [9]. A 
114 p-value of $\leq 0.001$ and an absolute fold-difference between venous and capillary or

115 between pre- and post-vaccination in expression of greater than 1.25 was used to

116 select differentially expressed transcripts. To find total genes differentially expressed

117 at baseline, the total numbers from $-7 \mathrm{~d}$ and $0 \mathrm{~d}$ were added and replicates removed,

118 whilst transcripts consistently differentially expressed were calculated using genes

119 differentially expressed at both $-7 \mathrm{~d}$ and $0 \mathrm{~d}$. To compare numbers of transcripts

120 between venous and capillary samples, a McNemar's test was performed. Bland-

121 Altman plots containing all genes differentially expressed post-vaccination were used

122 to examine whether the transcript expression changes were comparable when

123 measured in venous and capillary blood.

\section{Pathway Analysis}

125 Pathway analysis was performed on differentially expressed transcripts using the 126 publically available analysis tool InnateDB [10]. Pathways were considered 127 differentially expressed if the false-discovery rate was $<0.05$. Two pathway analyses 128 were performed at baseline: an analysis using total genes differentially expressed 129 between venous and capillary blood, and a split analysis using only the genes higher 130 in either venous or capillary blood.

132 Gene Set Enrichment Analysis

133 Gene Set Enrichment Analysis (GSEA) was performed using R. Gene lists were 134 ranked by $\log 2$ FC and analysed for enrichment of blood transcriptional modules 135 (BTMs) previously described by Li et al [11, 12]. Nominal p-values for Normalised 136 Enrichment Scores (NES) were calculated using 1000 random gene set iterations and 137 corrected for multiple testing using the Benjamini-Hochberg (BH) method. Gene lists 138 used included the comparisons of capillary against venous at baseline (day 0) and 139 capillary against venous fold change after vaccination. 


\section{Results}

\section{Investigation of Variability in Expression}

144 To examine the variability in expression levels at a global level between venous 145 and capillary samples, numbers of detected probes, mean probe intensity 146 distributions, and distributions of variance were produced (Supplementary 147 figure 1). These showed that while there was a higher number of probes detected 148 on average in venous than capillary samples, variance and mean probe intensity 149 were similar between venous and capillary blood.

\section{Comparison of Venous and Capillary samples at Baseline}

151 At baseline (-7d and 0d), there were a total of 6641 transcripts with differential 152 expression in capillary compared to venous samples, of which 4940 were consistent 153 between the two baseline time points (See Figure 1A). Expression of 4095 transcripts 154 were higher in venous blood, with 2984 consistent between the two timepoints, whilst 1552546 were higher in capillary blood, with 1956 consistent between the two timepoints. 156 Regression analysis from -7d to 0d showed no statistically significant differences, so 157 0d was subsequently used as baseline for pathway analysis.

Differences at 0d corresponded to 100 pathways that were differentially expressed between venous and capillary blood (Supplementary figure 2A). The

160 majority of the pathways with differential enrichment, between venous and capillary 161 blood, were upregulated in venous blood; only five pathways significantly were 162 enriched in capillary compared blood, including the mRNA splicing and processing, 163 gene expression - which encompasses transcription, mRNA processing and 164 translation - and immune system pathways - which encompasses innate and adaptive 165 immune system along with cytokine signalling (See Figure 1B). Those greater in 166 venous blood include pathways representing the spliceosome, nonsense mediated 167 decay, formation of a pool of free 40S subunits, GTP hydrolysis and joining of 60S 168 subunit, cap-dependent translation initiation, eukaryotic translation, initiation and 169 elongation (See Figure 1C). These pathways are sequentially active in the processing 170 of RNA, post-transcriptional regulation and translation of proteins [13-16]. There 171 were a number of other metabolic pathways differentially enriched, including 172 mitochondrial translational pathways and those involved in organelle biogenesis and 173 maintenance. The immune related pathways included Class I MHC mediated antigen 
174 processing and presentation, antigen processing (ubiquitination and degradation),

175 adaptive immune system - which includes T- and B-cell receptor signalling, major 176 histocompatibility complex function, and other immune related signalling - and toll177 like receptor (TLR) 2, 3, 4 and 6 cascades (See Figure 1D). Additionally, in the blood 178 transcriptional module (BTM) analysis, modules involved in generic immune 179 activation and CD4 lymphocyte cell cycle have greater enrichment in venous than 180 capillary (Supplementary figure 2B).

181 These results were underlined by the different clustering behaviour between the 182 samples when performing principal component analysis (PCA) (Supplementary figure 183 3), with $37 \%$ of the gene expression variance accounted for by the first principal 184 component. This analysis showed some separation by batch on the second principal 185 component (Supplementary figure 4), but one batch was split between venous and 186 capillary, and separated on the first principal component. This showed that the batch 187 effect does not appear to impact on the separation caused by the difference between 188 venous and capillary samples.

\section{See Figure 1}

\section{Comparison of Venous and Capillary Fold-Change (FC) Post Vaccination}

192 At 12 hours following influenza vaccination a total of five transcripts from the 193 capillary samples were differentially expressed (DE) when compared with baseline, 194 compared with 76 transcripts in the venous samples when compared with baseline. At 19524 hours there were nine transcripts differentially expressed compared with baseline 196 in the capillary samples, and 277 transcripts in the venous samples (Supplementary 197 figure 5 and Supplementary figure 6). For the pneumococcal vaccine samples, at 12 198 hours a total of 239 transcripts from the capillary samples had a differential 199 expression compared to baseline, compared to 269 transcripts differentially expressed 200 in the venous samples. At 24 hours there were 70 transcripts differentially expressed 201 compared to baseline in the capillary samples, and 280 transcripts in the venous 202 samples (Table 2). A McNemar's test highlighted the disparities in differential gene 203 expression findings in venous, compared with capillary, samples (influenza 12h: p204 value $=7.381 \times 10^{-15}$, influenza $24 \mathrm{~h}$ : $\mathrm{p}$-value $<2.2 \times 10^{-16}$, pneumococcal $12 \mathrm{~h}$ : $\mathrm{p}$-value $=$ 205 0.1389, pneumococcal 24h: p-value $<2.2 \times 10^{-16}$ ). 
207 Correlation plots produced showed the general relationship between venous and 208 capillary expression (Supplementary figure 7). However, Bland-Altman plots of the 209 difference between transcript FC (DE transcripts only) measured in venous and 210 capillary blood compared with the mean FC of these two sampling methods showed a 211 lack of agreement, with an evident relationship between the difference in the FC 212 measures of these methods and their mean (See Figure 2). In the influenza vaccine 213 groups there was a moderate negative trend of differences, proportional to mean of the 214 two measurements. Moreover, 24h after influenza vaccine the average of the 215 differences between the methods was less than zero; meaning for these transcripts, on 216 average, the log FC measured in capillary blood was higher than that measured in 217 venous blood. Conversely, for the pneumococcal vaccine, there was a moderate 218 positive trend of differences, with reference to mean of the two measurements; and for 219 both $12 \mathrm{~h}$ and $24 \mathrm{~h}$ after vaccination the average of the differences between the 220 methods was more than zero, indicating the average log FC in venous blood was 221 higher than that measured in capillary blood (See Figure 2).

222 Multiple different biological pathways were enriched in fold-changes of 223 transcript expression from baseline (Supplementary figure 8). There were a greater 224 number of pathways differentially expressed in the venous than the capillary groups. 225 This was true for both vaccines and at all times after vaccination. There were multiple 226 immune pathways differentially expressed from baseline in the venous groups (more 227 extensively analysed by Obermoser et al.)[7]. 
230 Comparison of Fold-Changes Between Venous and Capillary samples

231 Multiple genes had different fold-changes between venous and capillary samples 232 (Table 3). There were multiple blood transcriptional modules (BTMs) differentially 233 enriched between capillary and venous samples (Figure 3). At both $12 \mathrm{~h}$ and $24 \mathrm{~h}$ after 234 vaccination with the influenza vaccination, the BTMs enriched in capillary blood 235 represented T-cell activation and NK cell related patterns. In contrast, responses in 236 venous blood were characterized by enrichment in monocyte and generic immune 237 activation modules (Figure 3A and 3C). In contrast, while no BTMs were enriched in 238 venous blood 12hrs following pneumococcal vaccination, capillary samples showed a 239 moderate pattern of DCs, monocytes and interferon related signatures (Figure 3B). 240 The responses were increased 24hrs after pneumococcal vaccination with 241 inflammatory signals, monocytes, cell cycle and interferon responses strongly 242 enriched in capillary blood, where as T and NK cell signatures were enriched in 243 venous blood (Figure 3D).

244 A pathway over-representation analysis was also performed, using the differentially 245 expressed genes detailed above. At 24h after pneumococcal or influenza vaccinations 246 there were no pathways differentially expressed. At $12 \mathrm{~h}$ after vaccination, there were 247 multiple pathways enriched by differentially expressed genes. After influenza 248 vaccination, these included multiple immune pathways, including interferon $\alpha / \beta$ 249 signalling, multiple toll-like receptor (TLR) related pathways including activated 250 TLR3 and TLR4 signalling. Furthermore, multiple pathways related to mRNA 251 transcript expression, including translational and transcriptional pathways, along with non-sense mediated decay pathways, had differential fold changes from baseline 253 between venous and capillary blood (Supplementary figure 8A). Similarly, multiple 254 pathways had differential fold changes following pneumococcal vaccination including 255 immune pathways, cell trafficking and membrane trafficking pathways 256 (Supplementary figure 8B).

\section{Table 3:}

\section{See Figure 3}


263 This study demonstrates a clear difference in gene expression between venous and 264 capillary blood samples, both before and after vaccination with influenza and 265 pneumococcal vaccines. Depending on which sample type is utilised, inferences about 266 the molecular mechanisms of immunity may be different, leading to different 267 conclusions regarding pathways to the most robust vaccine response and potentially 268 modifying future vaccine development work. While vaccines are one of the most 269 important public health interventions, we have limited understanding of the molecular 270 mechanisms underpinning the development of vaccine-induced protection. High271 throughput technologies enable investigation of the detailed responses to vaccination, 272 yet issues such as standardisation exist, potentially leading to confounded or 273 erroneous conclusions. Based on these analyses, further data directly comparing 274 venous and capillary samples are required to enable future standardisation of sample 275 type, which would help future study design.

276 At baseline, there were 4940 genes consistently differentially expressed between 277 venous and capillary blood, with a majority of these higher in venous than capillary 278 blood. This corresponds to 94 pathways that are enriched for genes with greater 279 expression in venous blood, including a number of important translation-related, 280 immune and cell trafficking pathways. However, only 2 blood transcriptional modules 281 were differentially enriched between venous and capillary blood, with both modules, 282 one involved in generic immune activation, the other in CD4 cell cycle, enriched in 283 venous blood.

284 After vaccination, there were a greater number of genes differentially expressed in the 285 venous than the capillary samples, possibly reflecting the difference in sample sizes of 286 these groups. After influenza vaccination, there was enrichment of BTMs involved 287 with T-cell proliferation and activation, and NK cell related modules in capillary 288 blood. In venous blood, there was enrichment of monocyte and generic immune 289 module activation modules. After pneumococcal vaccination, there was enrichment of 290 interferon, dendritic cell, inflammatory and monocyte signatures in capillary blood. 291 After 24h, there was also enrichment of T and NK cell signatures in venous blood. At 292 all time points except $12 \mathrm{~h}$ after influenza vaccination, interferon activation was 293 enriched in capillary blood.

294 Similarly, after influenza vaccination, there were a number of interferon related 295 pathways differentially expressed between venous and capillary blood. After 
pneumococcal vaccination, the pathways included Fc $\gamma \mathrm{R}$ dependent phagocytosis, along with multiple pathways involved with cellular response to bacterial infection. Obermoser et al., found that interferon signalling was one of the most important changes in the first 48 hours after vaccination with the trivalent inactivated influenza vaccine, as did Cao et al. and Nakaya et al., again with the trivalent inactivated influenza vaccine $[3,7,17]$. Obermoser et al. also highlighted modules M5.1 and

302 M4.3 as among those having the greatest enrichment at 1 day after vaccination. M5.1

303 - involved in T cell activation and signalling - was higher in venous blood at 24h 304 after pneumococcal vaccination, while M4.3 - involved in myeloid cell receptors and transporters - was higher in capillary blood at $24 \mathrm{~h}$ after pneumococcal vaccination, 306 but higher in venous blood at $24 \mathrm{~h}$ after influenza vaccination [7]. This is important, as 307 it shows that one of the main differences in gene expression changes from baseline 308 between venous and capillary samples is in a gene set key that has been used to 309 characterise the immunogenicity of the influenza vaccine. Additionally, Obermoser et al. noted that one of the key changes after pneumococcal vaccination is in FcyR 311 signalling, which is critical in the development of protective immunity against 312 Streptococcus pneumoniae, via opsonophagocytosis [7].

313 A key question is whether the differences we observed, between venous and capillary 314 blood, reflect real biological differences in gene expression between these blood 315 sources, or whether these findings could be explained by sampling artefact. 316 Physiologically, capillary blood is different from venous blood, with different levels of proteins, enzymatic activity and electrolytes, along with different changes after 319 Several studies have demonstrated that capillary and venous blood have different 320 white blood cell counts, with capillary blood showing a higher leukocyte count than 321 venous blood [22, 23]. Additionally, there is some evidence that venous and capillary 322 blood are immunologically different, with higher levels of TNF- $\alpha$ in capillary blood at 323 baseline and after stimulation, along with differences in the level of IL-10 [24, 25].

324 It is also possible that differences could be caused by the physical method of blood 325 extraction leading to different gene expression, resulting in artefactual differences. 326 However, it has been shown that the minimum time between the stress stimulus and 327 mRNA production is greater than 15 minutes, longer than it would take for cell lysis 328 after blood taking. Whilst capillary blood yields less RNA than venous blood, it 329 produces equivalent quality RNA [26, 27]. 
330 This study is important in illuminating factors to be taken into account in designing

331 future studies testing the immunogenicity of vaccines. Fingerprick blood samples can

332 be taken more regularly, without a trained professional, and it is straightforward to

333 carry out longitudinal, frequent sampling within the same individual. In contrast,

334 obtaining venous blood requires a trained phlebotomist and can only be taken with

335 limited frequency. In addition, fingerprick (or heelprick) samples are much less

336 problematic in paediatric studies. This means that the findings presented in this paper

337 have an important effect on the design of studies into the immunogenicity of vaccines

338 in the paediatric setting.

339 There are some limitations to this study. The sample size used was small, reducing the

340 ability to detect differences, and sample group sizes differed within the study. There is,

341 additionally, a lack of corroborating data, and the study was not originally designed to

342 illuminate causal processes, making causation difficult to ascertain.

343 This is a novel investigation, as no group has previously used this approach to

344 compare venous and capillary blood, and yields results significant both for the basic

345 physiological understanding of differences in gene expression, and for the design of

346 future trials, as we show that capillary and venous blood were not equivalent for the

347 investigation of gene expression changes post vaccination.

348 However, with comparisons at only four time-points, further investigation is vital. The

349 ease of taking fingerprick samples makes it important that subsequent papers add

350 fingerprick samples to their study protocol and compare them with venous samples to

351 further investigate these differences, specifically pre- and post vaccination at multiple

352 time points.

353

354 Acknowledgement

355 We would like to acknowledge Damien Chaussabel for making the dataset

356 (GSE30101) described in this article publicly available on the Gene Expression

357 Omnibus database, without which this analysis would not have been possible. 


\section{Authors' contributions}

360 DFS conducted the analysis presented in this manuscript, with input from CJB, MS, 361 and DOC. DFS, DOC and AJP contributed to the conception and design of this work. 362 All authors contributed to the interpretation and drafting of this manuscript.

\section{Funding statement}

366 AJP is a Jenner Investigator and James Martin senior fellow. The authors received no 367 financial support or other form of compensation related to the development of the 368 manuscript.

\section{Conflict of interests}

371 The authors report no conflict of interests. 
373 1. O'Connor D, Pollard AJ: Characterizing vaccine responses using host

2. Querec T, Akondy R, Lee E, Cao W, Nakaya H, Teuwen D, Pirani A, Gernert $\mathrm{K}$, Deng J, Marzolf $\mathrm{B}$, et al: Systems biology approach predicts immunogenicity of the yellow fever vaccine in humans. Nat Immunol 2009, 10:116-125.

3. Nakaya HI, Wrammert J, Lee EK, Racioppi L, Marie-Kunze S, Haining WN, Means AR, Kasturi SP, Khan N, Li GM, et al: Systems biology of vaccination for seasonal influenza in humans. Nat Immunol 2011, 12:786-795.

4. Nakaya HI, Clutterbuck E, Kazmin D, Wang L, Cortese M, Bosinger SE, Patel NB, Zak DE, Aderem A, Dong T, et al: Systems biology of immunity to MF59-adjuvanted versus nonadjuvanted trivalent seasonal influenza vaccines in early childhood. Proc Natl Acad Sci U S A 2016.

5. Haining $\mathrm{WN}$, Wherry EJ: Integrating genomic signatures for immunologic discovery. Immunity 2010, 32:152-161.

6. Bucasas KL, Franco LM, Shaw CA, Bray MS, Wells JM, Niño D, Arden N, Quarles JM, Couch RB, Belmont JW: Early patterns of gene expression correlate with the humoral immune response to influenza vaccination in humans. J Infect Dis 2011, 203:921-929.

7. Obermoser G, Presnell S, Domico K, Xu H, Wang Y, Anguiano E, ThompsonSnipes L, Ranganathan R, Zeitner B, Bjork A, et al: Systems scale interactive exploration reveals quantitative and qualitative differences in response to influenza and pneumococcal vaccines. Immunity 2013, 38:831-844.

8. $\quad \mathrm{Du} \mathrm{P}, \mathrm{Kibbe} \mathrm{WA}$, Lin SM: lumi: a pipeline for processing Illumina microarray. Bioinformatics 2008, 24:1547-1548.

9. Ritchie ME, Phipson B, Wu D, Hu Y, Law CW, Shi W, Smyth GK: limma powers differential expression analyses for RNA-sequencing and microarray studies. Nucleic Acids Res 2015, 43:e47.

10. Breuer K, Foroushani AK, Laird MR, Chen C, Sribnaia A, Lo R, Winsor GL, Hancock RE, Brinkman FS, Lynn DJ: InnateDB: systems biology of innate immunity and beyond--recent updates and continuing curation. Nucleic Acids Res 2013, 41:D1228-1233.

11. Subramanian A, Tamayo P, Mootha VK, Mukherjee S, Ebert BL, Gillette MA, Paulovich A, Pomeroy SL, Golub TR, Lander ES, Mesirov JP: Gene set enrichment analysis: a knowledge-based approach for interpreting genome-wide expression profiles. Proc Natl Acad Sci U S A 2005, 102:15545-15550.

12. Li S, Rouphael N, Duraisingham S, Romero-Steiner S, Presnell S, Davis C, Schmidt DS, Johnson SE, Milton A, Rajam G, et al: Molecular signatures of antibody responses derived from a systems biology study of five human vaccines. Nat Immunol 2013.

13. Warnasooriya C, Rueda D: Single-molecule fluorescence-based studies on the dynamics, assembly and catalytic mechanism of the spliceosome. Biochem Soc Trans 2014, 42:1211-1218. 
14. Metze S, Herzog VA, Ruepp MD, Mühlemann O: Comparison of EJCenhanced and EJC-independent NMD in human cells reveals two partially redundant degradation pathways. RNA 2013, 19:1432-1448.

15. Martínez-Salas E, Lozano G, Fernandez-Chamorro J, Francisco-Velilla R, Galan A, Diaz R: RNA-binding proteins impacting on internal initiation of translation. Int J Mol Sci 2013, 14:21705-21726.

16. Hinnebusch AG: The scanning mechanism of eukaryotic translation initiation. Annu Rev Biochem 2014, 83:779-812.

17. Cao RG, Suarez NM, Obermoser G, Lopez SM, Flano E, Mertz SE, Albrecht RA, García-Sastre A, Mejias A, Xu H, et al: Differences in antibody responses between trivalent inactivated influenza vaccine and live attenuated influenza vaccine correlate with the kinetics and magnitude of interferon signaling in children. J Infect Dis 2014, 210:224-233.

18. Kupke IR, Kather B, Zeugner S: On the composition of capillary and venous blood serum. Clin Chim Acta 1981, 112:177-185.

19. Wurzinger S, Bratu M, Wonisch W, Wintersteiger R, Halwachs-Baumann G, Porta S: Interdependency of the oxidizability of lipoproteins and peroxidase activity with base excess, $\mathrm{HCO3}, \mathrm{pH}$ and magnesium in human venous and capillary blood. Life Sci 2006, 78:1754-1759.

20. Wittbrodt MT, Espinoza S, Millard-Stafford ML: Biological variation of plasma osmolality obtained with capillary versus venous blood. Clin Chem Lab Med 2015, 53:1613-1619.

21. Colagiuri S, Sandbaek A, Carstensen B, Christensen J, Glumer C, Lauritzen T, Borch-Johnsen K: Comparability of venous and capillary glucose measurements in blood. Diabet Med 2003, 20:953-956.

22. Yang ZW, Yang SH, Chen L, Qu J, Zhu J, Tang Z: Comparison of blood counts in venous, fingertip and arterial blood and their measurement variation. Clin Lab Haematol 2001, 23:155-159.

23. Schalk E, Heim MU, Koenigsmann M, Jentsch-Ullrich K: Use of capillary blood count parameters in adults. Vox Sang 2007, 93:348-353.

24. Eriksson M, Sartono E, Martins CL, Balé C, Garly ML, Whittle H, Aaby P, Pedersen BK, Yazdanbakhsh M, Erikstrup C, Benn CS: A comparison of ex vivo cytokine production in venous and capillary blood. Clin Exp Immunol 2007, 150:469-476.

25. Faulkner SH, Spilsbury KL, Harvey J, Jackson A, Huang J, Platt M, Tok A, Nimmo MA: The detection and measurement of interleukin-6 in venous and capillary blood samples, and in sweat collected at rest and during exercise. Eur J Appl Physiol 2014, 114:1207-1216.

26. Arner E, Daub CO, Vitting-Seerup K, Andersson R, Lilje B, Drabløs F, Lennartsson A, Rönnerblad M, Hrydziuszko O, Vitezic M, et al: Transcribed enhancers lead waves of coordinated transcription in transitioning mammalian cells. Science 2015, 347:1010-1014.

27. Robison EH, Mondala TS, Williams AR, Head SR, Salomon DR, Kurian SM: Whole genome transcript profiling from fingerstick blood samples: a comparison and feasibility study. BMC Genomics 2009, 10:617. 
475

476

477

478

Supplementary

479

480

See Supplementary figure 1

481 See Supplementary figure 2:

482 See Supplementary figure 3: 